Changes in cal bi ndi $n$ - D28k and par val bum $n$ expression in the superi or ol i vary compl ex fol I owing uni I ateral cochl ear abl at i on in neonat al $r$ ats

\begin{tabular}{|l|l|}
\hline 著者 & Hat ano M yako, Fur ukawa M t sur u, I t o Makot o \\
\hline $\begin{array}{l}\text { j our nal or } \\
\text { publ i cat i on ti tl e }\end{array}$ & Act a Ot o- Lar yngol ogi ca \\
\hline vol une & 129 \\
\hline nunber & 8 \\
\hline page r ange & $839-845$ \\
\hline year & 2009- 01-01 \\
\hline URL & ht t p: //hdl . handl e. net /2297/19812 \\
\hline
\end{tabular}




\title{
Changes in calbindin-D28k and parvalbumin expression in the superior olivary complex following unilateral cochlear ablation in neonatal rats
}

\author{
Hatano, Miyako MD, PhD, Furukawa MD, PhD, Mitsuru, Ito, Makoto MD, PhD \\ Department of Otolaryngology-Head and Neck Surgery, Kanazawa University, \\ Graduate School of Medical Science, 13-1 Takaramachi, Kanazawa, Ishikawa, 920-8640, Japan
}

\begin{abstract}
Conclusion: Unilateral congenital deafness with a volume reduction in cochlear nucleus $(\mathrm{CN})$ induced changes in the Calcium-binding proteins (CaBPs) in the contralateral superior olivary complex (SOC) in rat. With the loss of neurons and a volume reduction in the $\mathrm{CN}$, a decrease in the input to the contralateral SOC may occur, which results in the downregulation of CaBPs in these nuclei. This study may provide some implications regarding the neurochemistry in the auditory brainstem of deaf children.

Objectives: Hearing loss produced by cochlear damage during early development can result in persistent changes in the organization of the central auditory system in adults. The purpose of the present study was to investigate the neurochemical changes produced in the auditory brainstem of rats with unilateral cochlear ablation conducted prior to the onset of hearing.

Methods: Following unilateral cochlear ablation during early development, we examined the changes in the distribution of two CaBPs, calbindin-D28k (CB) and parvalbumin (PV), in the SOC.

Results: Upon reaching adulthood, a marked decrease in CB and PV-immunoreactive neurons was observed in the contralateral SOC, particularly in the medial nucleus of the trapezoid body (MNTB), although no neuronal cell death was observed. A volume reduction in the ipsilateral $\mathrm{CN}$ was also observed.
\end{abstract}

Key Words: Unilateral cochlear ablation, development, superior olivary complex (SOC), cochlear nucleus $(\mathrm{CN})$, calcium-binding protein

\section{Abbreviations}

SOC, superior olivary complex; MNTB, medial nucleus of the trapezoid body; LSO, lateral superior olive; MSO, medial superior olive; SPN, superior periolivary nucleus; $\mathrm{CN}$, cochlear nucleus; AVCN, anterior ventral cochlear nucleus; PVCN, posterior ventral cochlear nucleus; DCN, dorsal cochlear nucleus; CaBPs, calcium-binding proteins; CB, calbindin-D28k; PV, parvalbumin; ABR, auditory brainstem response 


\section{Inroduction}

Brain plasticity is a complex property that undergoes massive developmental changes after birth. In general, input deprivation in the nervous system causes functional and structural changes through expression of neural plasticity. Hearing loss produced by cochlear damage during early development can result in dynamic changes in the organization of the central auditory structures in adults. These changes include morphological and neurochemical changes in neurons, re-routing of neural connections, and abnormal physiological responses of the neurons in the auditory hindbrain, midbrain and forebrain. For example, congenital deafness results in physiological and anatomical transneural changes in auditory brainstem nuclei [1]. In gerbils, following early unilateral cochlear lesions, neurons in the ventral cochlear nucleus (VCN) on the side of the lesion establish abnormal connections with the superior olivary complex (SOC), including inappropriate targets within the ipsilateral medial nucleus of the trapezoid body (MNTB), bilateral medial superior olive (MSO) and the contralateral lateral superior olive (LSO) $[1,2]$. Other studies of animal models of deafness have shown abnormal synaptic terminals of the auditory nerve [3]. Despite the many morphological studies that have investigated the changes in the central auditory system caused by cochlear ablation before the onset of hearing, the neurochemical plasticity still remains unknown. In addition, there have been no reported studies that have examined the changes in the neurotransmitter release in animals with cochlear damage.

Calbindin D-28k (CB) and parvalbumin (PV) are widely expressed in the brainstem auditory nuclei $[4,5] . \quad \mathrm{CB}$ and PV are calcium-binding proteins (CaBPs) that exist in distinct subpopulations of neurons within the central nervous system [6]. The pattern of CB immunostaining in the SOC and $\mathrm{CN}$ has been well characterized in rat, guinea pig, and cat [4, 7]. All of these studies have reported that $\mathrm{CB}$ immunoreactivity is present in every principal nucleus within the SOC. In addition, all nuclei of the SOC contained abundant PV-immunoreactive elements [8]. The pattern of PV immunostaining in the SOC also has been well characterized in rat and guinea pig [8]. CaBPs are involved in the release of neurotransmitters, the functioning of ion channels in the neuronal membranes, and the regulation of enzyme activities at pre- and postsynaptic sites and during synaptic short-time plasticity [9]. They are sequentially expressed during the auditory brainstem development. Upon maturation, CB is drastically reduced or entirely lost in many auditory nuclei, while PV appears relatively late in the somata and then rapidly increases to adult levels. During neuronal development, it is believed that CaBPs could be involved in buffering the intracellular free $\mathrm{Ca} 2+$ concentration, which may be necessary for modification of synaptic 
efficiency $[4,8]$.

In the auditory pathway, unilateral deaf models are frequently used to identify the effects of monaural hearing loss, as this allows for straightforward comparisons to be made between the uptakes in both the normal and deprived sides within the same animal. However, in the rat auditory system ontogeny, there have been few systemic studies that examined the neurons containing CaBPs $[4,8]$. In the present study, we investigated the changes in the $\mathrm{CaBP}$ expressions within the auditory brainstem in an animal model with congenital deafness. Unilateral cochlear ablations before the onset of hearing have been used as models of congenital deafness. This study may provide some implications regarding the neurochemistry in the auditory brainstem of deaf children.

\section{Materials and methods}

\section{Animals and Surgical Procedures}

A total of 10 adult male and female Sprague-Dawley rats were used. The Animal Research Committee of Kanazawa University Graduate School of Medical Science approved the care and use of the animals in the present study. Postnatal day 0 (P0) to P1 newborn rats in the experimental group $(n=7)$ were anesthetized by hypothermia and a skin incision was made inferior to the right pinna. The lateral semicircular canal and middle ear soft tissue were exposed by blunt dissection, taking care to avoid injury to the facial motor nerve. After removing the stapes, a syringe needle was inserted through the oval window into the cochlea and the cochlea contents were aspirated. The skin incision was closed with glue. The newborn rats were allowed to recover under a heating lamp and returned to their mother after recovery. In addition to these 7 experimental animals, three normal, 4-month-old rats served as unmanipulated controls.

\section{Hearing Loss Assessment}

Auditory function was assessed by recording auditory brainstem response (ABR). The measurements of ABR thresholds were performed at frequencies of 4, 8 and $16 \mathrm{kHz}$ after a post-surgical survival time of 4 months. Animals were anesthetized with pentobarbital sodium (50 mg/kg ip) and kept warm with a heating pad. Thresholds at each frequency were verified at least twice. An 80-dB shift from the normal control threshold averaged across the three frequencies was considered to represent deafness.

Tissue Processing

After ABR assessment, the deaf animals were immediately given an injection 
of pentobarbital sodium (120 mg/kg ip) and perfused transcardially with phosphate-buffered saline followed by $4 \%$ paraformaldehyde. The brains were removed and post-fixed in the same fixative overnight and stored in a $30 \%$ sucrose buffer for several days, and cut serially at $40 \mu \mathrm{m}$ in the frontal plane on a freezing microtome (Leica SM 2000R). Typically each brain was cut into three alternating series of section, one of which was used for counterstaining with cresyl violet and the other two were analyzed by immunocytochemistry.

\section{$C B$ and PV Immunocytochemistry}

Free-floating sections were incubated in $0.03 \% \mathrm{H}_{2} \mathrm{O}_{2}$ for 10 min to block unspecific peroxidase activity. Sections were then washed with phosphate-buffered saline (0.1 M; PBS) with $0.2 \%$ Triton X-100 (PBST) and incubated with a primary antibody overnight at room temperature. The primary antibody was either a mouse monoclonal antibody against CB (Sigma, St. Louis, MO, USA; clone CB-955; dilution of 1:12000) or a mouse monoclonal antibody against PV (Sigma; clone PA-235; dilution of 1:30000). The primary antibodies were detected using the EnVision+ System (DakoCytomation California, Carpinteria, CA, USA). Sections were incubated with the 3,3'-diaminobenzidine (DAB) substrate-chromagen solution (EnVision+ System) for 5 min. Finally, sections were washed in PBS and then mounted on gelatin-coated slides.

\section{Analysis of MNTB}

Sections were examined by bright-field microscopy using either a $\times 40$ or a $\times$ 60 lens (Nikon Eclipse E1000 microscope; Nikon, Auckland, New Zealand), with images captured using a Nikon digital camera DXM1200C (Nikon). Labeled neurons in the MNTB were counted on the cresyl violet and immunocytochemical sections. For a neuron to be counted in the cresyl violet section, a well-defined cytoplasm and nuclear outline with a clearly visible nucleolus had to be present. Neuron counts were obtained from the MNTB on both sides of the brainstem. Immunocytochemically positive cells were characterized by the orange/brown DAB labeling, whereas negative cells exhibited no labeling. Cells were considered to be immunopositive when they displayed stained brown somata.

\section{Optical Density Measurement}

The lesion-induced effects on $\mathrm{CB}$ and PV expression were assessed by determination of the optical density in the superior paraolivary nucleus (SPN) and LSO using Image $\mathrm{J}$ software (NIH, Bethesda, MD, USA). For each animal, a mean operated and non-operated optical density ratio was determined.

Measurements of Cochlear Nucleus Volume 
Using Image $\mathrm{J}$ software $(\mathrm{NIH})$, the perimeters of the $\mathrm{VCN}$ and the dorsal cochlear nucleus $(\mathrm{DCN})$ were traced $(10 \times$ objective $)$ from the cresyl violet sections. The total volume of each nucleus was then estimated from the total areas of the sections. Image Processing

To prepare the figures shown here, the size and brightness of each image was adjusted using Adobe Photoshop CS (Version 8.0.1). These adjustments in no way altered the immunostaining pattern of each antibody.

Statistical Analysis

All data are expressed as the mean \pm SD and were analyzed statistically by using a Student's t-test. Statistical significance was determined at the $\mathrm{p}<0.05$ level. The evaluation and data analyses were made by experimentally blind observers.

\section{Results}

No ABR waves were elicited at any frequency, even at $105 \mathrm{~dB}$ SPL, in cochlear ablated side, indicative of deafness. Although the area of the contralateral MNTB was about $60 \%$ to $80 \%$ of the size of the ipsilateral MNTB (Fig. 1A), neonatal cochlear ablation did not result in any loss of neurons in the MNTB (Figs. 1A and B, Table 1), as determined by cresyl violet staining. The total number of neurons in the MNTB was $1133.4 \pm 208.5$ on non-operated (left) and $1199.1 \pm 114.9$ on operated (right) side. In the controls, somata were positively stained for $\mathrm{CB}$ and $\mathrm{PV}$ in the MNTB, where virtually every CB or PV immunoreactive neuron was heavily labeled. In contrast to counts seen for the cresyl violet cells, there was a significant difference between the sides for the number of CB positive neurons in the MNTB, with $162.4 \pm$ 70.4 on non-operated (left) and $327.9 \pm 84.4$ on operated (right) side. (Figs. 1C and D, Table 1) The number of PV positive neurons in the MNTB also had significantly difference between the sides, with $151 \pm 96.3$ on non-operated (left) and $254.1 \pm 114.2$ on operated (right) side. With early cochlear ablation, there was a substantial reduction in the number of $\mathrm{CB}$ and PV immunopositive cells in the MNTB on the side that was contralateral to the ablation (Figs. 1E and F, Table 1).

----- Figure 1 and Table 1 Here-----

In contrast to the MNTB, in normal animals there were very few $\mathrm{CB}$ positive somata seen in the LSO and those were weakly stained. In the SPN, almost all immunoreactivity was restricted to the neuropil. There were no differences in the expression and staining of these proteins between the left and right sides in the control animals. As determined by optical density, there was a decrease in neuropil density observed in the contralateral LSO and SPN following unilateral cochlear damage during 
early development (Figs. 2A, B, Table 2). The mean operated (right) and non-operated (left) optical density ratio was $1.19 \pm 0.08$ (LSO) and $1.23 \pm 0.03$ (SPN).

All nuclei of the SOC contained abundant PV-immunoreactive elements in the normal animals. The highest density of PV-immunoreactive somata was found in the MNTB and the LSO. Many PV immunoreactive somata were also located in the SPN and in the periolivary areas. The neuropil in the whole SOC was very intensely PV immunoreactive. As demonstrated by optical density determinations, PV-immunoreactive cells in the SOC contralateral to the ablated side were less intensely labeled. The mean operated (right) and non-operated (left) optical density ratios were $1.23 \pm 0.11$ (LSO) and $1.30 \pm 0.07$ (SPN), respectively (Figs. 2C, D, Table 2).

-----Figure 2 and Table 2 Here-----

Cochlear ablation during early development is known to cause a reduction in the size of the cochlear nucleus ipsilateral to the lesion. We used Image $J$ in the present study and determined that as compared to each of the contralateral subdivisions of the $\mathrm{CN}$, the volumes of the AVCN, PVCN and DCN were reduced by $55.7 \pm 17.1 \%$, $62.1 \pm 17.4 \%$ and $21.5 \pm 10.2 \%$, respectively, (mean $\pm \mathrm{SD}$ ). Our results confirm previous reports on the $\mathrm{CN}$ volume reduction [10].

\section{Discussion}

Hearing loss produced by cochlear damage during early development can result in persistent changes in the organization of central auditory structures in adults. Although a number of previous studies have revealed changes in the morphology, projection and physiology of the central auditory pathway following cochlear ablation, the changes in neurotransmitter release have yet to be studied in detail [11]. CaBPs have been implicated in neurotransmitter release, and thus, immunostaining of these proteins might be used to follow the subtle changes that occur in neuronal activity. Since the presence of CaBPs is correlated with definite developmental stages of the central auditory system, CaBPs may also be the key to understanding neurotransmitter release in the mammalian auditory brainstem development.

Our results indicated that there were corresponding changes in the expression of the CaBPs, CB and PV, in the contralateral SOC nuclei of the rats subjected to early unilateral cochlear ablation, which suggests there were changes in regulation of the neurotransmitters in these structures. Cochlear ablation before the onset of hearing resulted in a decrease in the level of immunostaining of the CaBPs within the contralateral SOC, as compared to the ipsilateral side and staining seen for the control animals. The decrease of CaBPs may suggest that there is a decrease in the calcium 
concentration that is related to the decreased activity [12]. Our results suggest that there is downregulation of the neuroactivity in the contralateral SOC.

Another main finding in the present study was the occurrence of a substantial decrease in the number of $\mathrm{CB}$ and PV immunoreactive neurons, although there was no change noted in the total number of neurons in the MNTB following the unilateral cochlear ablation. The SOC is the initial site for the convergence of binaural input and is most likely involved in the localization of sounds [13]. As such, the SOC plays an important role in the formation of sensitivity to interaural comparisons of intensity and time for stimuli that reach the two ears. In the SOC, the MNTB supplies a major input to the ipsilateral LSO, relaying information from the contralateral CN. The LSO and MNTB have been reported to work as a single functional unit [14]. The MNTB assists this functional unit by providing the LSO with input from the contralateral ear [15]. Unilateral cochlear ablation reduces auditory inputs to the SOC and causes the outputs to be out of balance. However, the cresyl violet staining on both sides did not indicate there was any observable decrease in the number of the MNTB neurons. Additionally, between both sides in the control animals and for the lesioned side in ablated animals, there were no statistically significant differences noted for the number of $\mathrm{CB}$ and $\mathrm{PV}$ reactive cells in the MNTB. This finding implies that the immunoreactivity of the CaBPs for the MNTB in the ablated side is not affected by unilateral cochlear ablation during early development. It is possible that this could be because the MNTB on the ablated side is projected from the $\mathrm{CN}$ of the non-ablated side. There is also the possibility that the monaural input is responsible for the differences in the immunostaining of the CaBPs that was noted between the ipsilateral and contralateral SOC in the present study.

Matsubara et al. has suggested that $\mathrm{CB}$ is specific for "relay" neurons in a system that requires rapid, reliable transmission of impulses with minimal local processing or integration [7]. MNTB principal neurons most certainly receive secure synaptic input from the $\mathrm{CN}$ and these neurons have been reported to be intensely glycine-immunoreactive in the guinea pig [16]. There is strong evidence that glycine is a major inhibitory neurotransmitter in the mammalian central nervous system and additionally, the projection from the MNTB to the LSO is also considered to be inhibitory [17]. Our results suggest that the decrease in the immunoreactivity of the CaBPs is related to the inactivity of the inhibitory nervous system in the contralateral MNTB that occurs when there is unilateral cochlear ablation prior to the onset of hearing. We also observed a substantial decrease in $\mathrm{CB}$ immunoreactivity in the contralateral SPN and LSO, as well as in the contralateral MNTB. The PV 
immunostaining pattern that was observed following neonatal cochlear ablation in the SOC was similar to the CB immunostaining in this study.

Cochlear ablation leads to the loss of $\mathrm{CN}$ neurons in cases where ablation is performed during early development in rats and gerbils [10]. We also observed a volume loss in the CN, in particular for the AVCN and PVCN. There was a significant ipsilateral decrease in the volume of the VCNs, as compared with that seen for the DCN. This result suggests that the loss of neuronal connection from the $\mathrm{CN}$ to the contralateral MNTB causes a decrease in MNTB neuronal activity, even though it does not affect neuronal survival. In rat, projection to the MNTB comes from the contralateral globular/bushy cells of the VCN [18]. Born and Rubel were the first to describe the age-dependent change in the susceptibility of the avian $\mathrm{CN}$ neurons to deafferentation. Studies on mammals have indicated that the change in sensitivity to afferent deprivation in the mammalian VCN is temporally related to hearing onset [19]. In gerbil $\mathrm{CN}$, there is no loss of $\mathrm{CN}$ neurons observed following cochlear removal after $\mathrm{P} 7$, which is 5 days before the onset of hearing at P12. Mean volume reduction of the deafferented CN did not differ significantly between P11 and P93 [20]. There is the possibility that the downregulation of the CaBPs in the SOC might be caused by the loss of $\mathrm{CN}$ neurons. Further investigations using unilateral cochlear damage models without $\mathrm{CN}$ volume loss and cochlear ablation just before the onset of hearing will be required in order to clarify the real effect of the congenital hearing loss. Not only deafness, cochlear ablation itself, destructive deaf model, may have made effect our result. Congenital deaf model less damage of inner ear is more ideal for understanding human congenital deafness. Furthermore, although we used a unilateral deaf model of adult stage (4 months) of rats, to be able to understand the central auditory system of congenitally deaf children, a bilateral deaf model at earlier times needs to be examined.

\section{Acknowledgements}

This study was supported by a Grant-in-Aid for Scientific Research from the Ministry of Education, Culture, Sports, Science and Technology of Japan (\#19591961)

\section{References}

1. Russell FA, Moore DR: Afferent reorganisation within the superior olivary complex of the gerbil: development and induction by neonatal, unilateral cochlear removal. J Comp Neurol 1995;352:607-625.

2. Kitzes LM, Kageyama GH, Semple MN, Kil J: Development of ectopic projections from the ventral cochlear nucleus to the superior olivary complex induced 
by neonatal ablation of the contralateral cochlea. J Comp Neurol 1995;353:341-363.

3. Ryugo DK, Rosenbaum BT, Kim PJ, Niparko JK, Saada AA: Single unit recordings in the auditory nerve of congenitally deaf white cats: morphological correlates in the cochlea and cochlear nucleus. J Comp Neurol 1998;397:532-548.

4. Friauf E: Distribution of calcium-binding protein calbindin-D28k in the auditory system of adult and developing rats. J Comp Neurol 1994;349:193-211.

5. Celio MR: Calbindin D-28k and parvalbumin in the rat nervous system. Neuroscience 1990;35:375-475.

6. Andressen C, Blumcke I, Celio MR: Calcium-binding proteins: selective markers of nerve cells. Cell Tissue Res 1993;271:181-208.

7. Matsubara JA: Calbindin D-28K immunoreactivity in the cat's superior olivary complex. Brain Res 1990;508:353-357.

8. Lohmann C, Friauf E: Distribution of the calcium-binding proteins parvalbumin and calretinin in the auditory brainstem of adult and developing rats. $\mathrm{J}$ Comp Neurol 1996;367:90-109.

9. Caillard O, Moreno H, Schwaller B, Llano I, Celio MR, Marty A: Role of the calcium-binding protein parvalbumin in short-term synaptic plasticity. Proc Natl Acad Sci U S A 2000;97:13372-13377.

10. Moore DR, Rogers NJ, O'Leary SJ: Loss of cochlear nucleus neurons following aminoglycoside antibiotics or cochlear removal. Ann Otol Rhinol Laryngol 1998;107:337-343.

11. Oleskevich S, Walmsley B: Synaptic transmission in the auditory brainstem of normal and congenitally deaf mice. J Physiol 2002;540:447-455.

12. Celio MR, Heizmann CW: Calcium-binding protein parvalbumin as a neuronal marker. Nature 1981;293:300-302.

13. Masterton B, Jane JA, Diamond IT: Role of brainstem auditory structures in sound localization. I. Trapezoid body, superior olive, and lateral lemniscus. J Neurophysiol 1967;30:341-359.

14. Glendenning KK, Hutson KA, Nudo RJ, Masterton RB: Acoustic chiasm II: Anatomical basis of binaurality in lateral superior olive of cat. J Comp Neurol 1985;232:261-285.

15. Spangler KM, Warr WB, Henkel CK: The projections of principal cells of the medial nucleus of the trapezoid body in the cat. J Comp Neurol 1985;238:249-262.

16. Wenthold RJ, Huie D, Altschuler RA, Reeks KA: Glycine immunoreactivity localized in the cochlear nucleus and superior olivary complex. Neuroscience 1987;22:897-912. 
17. Moore MJ, Caspary DM: Strychnine blocks binaural inhibition in lateral superior olivary neurons. J Neurosci 1983;3:237-242.

18. Friauf E, Ostwald J: Divergent projections of physiologically characterized rat ventral cochlear nucleus neurons as shown by intra-axonal injection of horseradish peroxidase. Exp Brain Res 1988;73:263-284.

19. Born DE, Rubel EW: Afferent influences on brain stem auditory nuclei of the chicken: neuron number and size following cochlea removal. J Comp Neurol 1985;231:435-445.

20. Tierney TS, Russell FA, Moore DR: Susceptibility of developing cochlear nucleus neurons to deafferentation-induced death abruptly ends just before the onset of hearing. J Comp Neurol 1997;378:295-306.

\section{Figure legends}

Table 1; Numbers of neurons in the MNTB in control and ablated animals.

Table 2; Optical density values for CB and PV in the LSO and SPN after unilateral early cochlear ablation.

Figure 1; High magnification images illustrating the pattern of the cresyl violet stain (A, B), CB immunoreactivity (C, D) and PV immunoreactivity $(\mathbf{E}, \mathbf{F})$ in coronal sections of the MNTB in ablated rats. Scale bar $=100 \mu \mathrm{m}$ in B (applies to A - F)

Figure 2; Low magnification images illustrating the pattern of the $\mathrm{CB}$ immunoreactivity $(A, B)$ and PV immunoreactivity $(\mathbf{C}, \mathbf{D})$ in coronal sections of the LSO and the SPN in ablated rats. Scale bar $=200 \mu \mathrm{m}$ in B (applies to A - D) 
Table 1 Numbers of cells in MNTB in control and ablated animals

\begin{tabular}{|c|c|c|c|c|c|c|c|}
\hline & \multicolumn{3}{|c|}{ Left side (Contralateral) } & \multicolumn{3}{|c|}{ Right side (Ipsilateral) } & \multirow[b]{2}{*}{$\mathrm{n}$} \\
\hline & Cresyl violet & CB & PV & Cresyl violet & CB & PV & \\
\hline Control & $1190.3 \pm 100.9$ & $343 \pm 125.7$ & $210 \pm 35.4$ & $1207.3 \pm 231.5$ & $348 \pm 122.4$ & $201.3 \pm 41.2$ & 3 \\
\hline Right ablated & $1133.4 \pm 208.5$ & $162.4 \pm 70.4 *$ & $151 \pm 96.3$ & $1199.1 \pm 114.9$ & $327.9 \pm 84.4$ & $254.1 \pm 114.2$ & 7 \\
\hline
\end{tabular}

Values expressed are means and standard deviations. Asterisk $(*)$ indicates significant differences between sides in ablated animals. 
Table 2 Optical Density Values for CB and PV in LSO and SPN after unilateral cochlear ablation.

\begin{tabular}{lllll}
\hline & & Left side (Contralateral) & Right side (Ipsilateral) & Left / Right ratio \\
\hline CB & & & \\
& LSO & $219.5 \pm 5.4^{*}$ & $184.7 \pm 10.1$ & $1.19 \pm 0.08$ \\
& SPN & $213.5 \pm 6.0 *$ & $173.9 \pm 8.1$ & $1.23 \pm 0.03$ \\
PV & & & \\
& LSO & $138.5 \pm 6.7 *$ & $112.7 \pm 6.3$ & $1.23 \pm 0.11$ \\
& SPN & $169.0 \pm 7.9 *$ & $129.9 \pm 11.1$ & $1.30 \pm 0.07$ \\
\hline
\end{tabular}

Values expressed are means and standard deviations.

Asterisk $(*)$ indicates significant differences between sides; LSO, lateral superior olivary nucleus; SPN, superior paraolivary nucleus. 

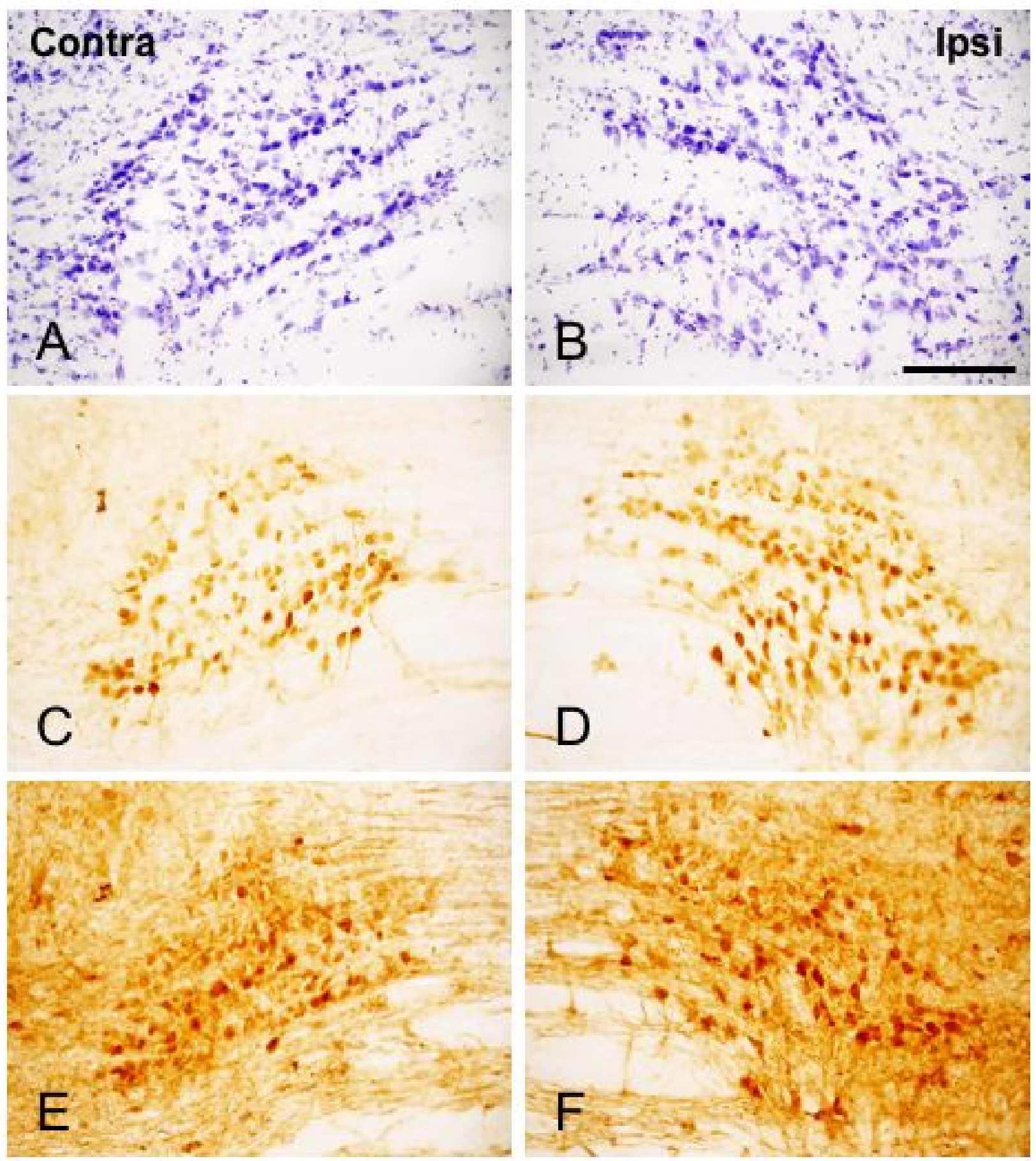

Fig.4 

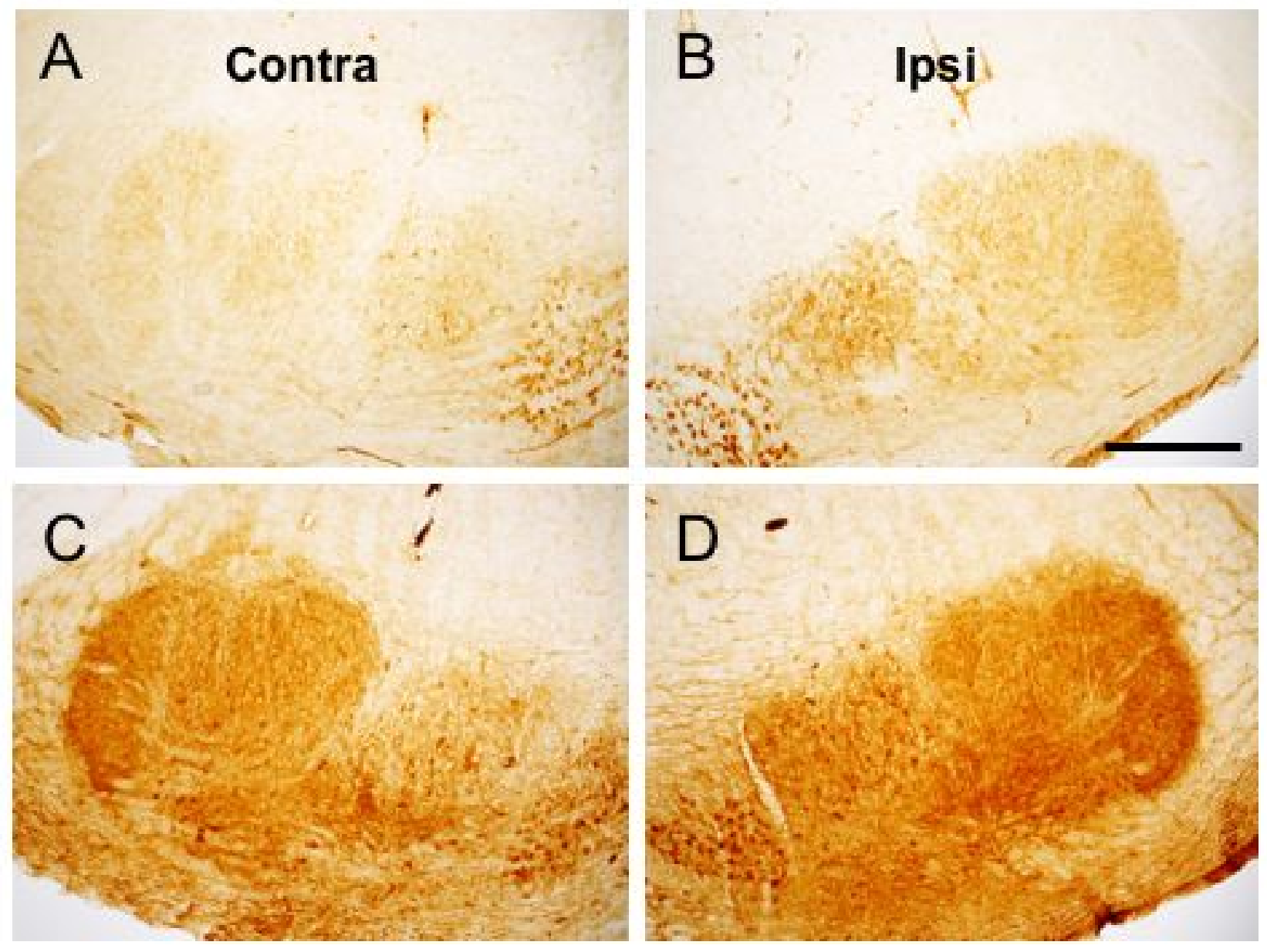

Fig.3 